\title{
Coincidental obstructive and post-hepatitic cholestatic jaundice
}

\author{
P. D. WeLSBY \\ M.B., M.R.C.P. \\ C. C. SMITH \\ M.B., M.R.C.P.
}

\author{
S. W. B. EWEN \\ M.B., Ph.D., M.R.C.Path. \\ P. W. BRUNT \\ M.D., F.R.C.P.
}

Departments of Medicine and Pathology, University of Aberdeen

\section{Introduction}

Obstructive jaundice due to carcinoma of the pancreas is uncommon in patients under the age of 40 , where jaundice is usually a sequel to viral hepatitis, drug ingestion, alcohol or gallstone disease. The appearance of extra-hepatic obstruction due to carcinoma during the cholestatic phase of viral hepatitis is unusual.

\section{Case report}

A previously healthy 37-year-old man was admitted to hospital with a 4-week history of jaundice, the inception of which followed a febrile illness with malaise, anorexia, and a distaste for cigarettes. During this period he developed dark urine and pale stools.

Two weeks after the appearance of jaundice he had developed generalized pruritus which persisted throughout his stay in hospital. There was no history of weight loss, abdominal or back pain and he denied alcohol abuse or exposure to drugs or industrial chemicals. There had been no known contact with jaundiced individuals, but 2 weeks after the appearance of jaundice in this patient his brother, whose food he prepared, developed an identical hepatitic illness. This brother subsequently died in hospital from fulminant hepatic failure; his $\mathbf{H B}_{\mathbf{s}} \mathbf{A g}$ was repeatedly negative, but percutaneous Menghini liver biopsy taken pre-terminally had shown the features of acute post-infective hepatic necrosis.

Examination at the time of admission revealed a lucid, cheerful, fit-looking but deeply jaundiced man. There were no stigmata of chronic liver disease or of hepatic pre-coma. Widespread excoriations were present from scratching. His liver was firm, tender and smoothly enlarged two finger breadths below the costal margin, and the spleen was easily palpable. Physical examination revealed no other abnormality save for a sinus bradycardia.

His haemoglobin was $14.2 \mathrm{~g} / \mathrm{dl}$; WBC $0.3 \times 10^{9} / 1$ (76\% neutrophils, $20 \%$ lymphocytes, $4 \%$ mono- cytes). The blood film revealed anisocytosis and target cells in addition to the leucopenia. The serum bilirubin was $210 \mathrm{mmol} / \mathrm{l}$; alkaline phosphatase 340 i.u./l; SGOT 65 i.u./1; SGPT 56 i.u./1; and prothrombin time (BCR) normal. His blood urea was $4 \mathrm{mmol} / 1$; potassium $3.2 \mathrm{mmol} / 1$; sodium 141 $\mathrm{mmol} / \mathrm{l}$; chloride $96 \mathrm{mmol} / \mathrm{l}$; and serum cholesterol $13.6 \mathrm{mmol} / \mathrm{l}$. The $\mathrm{HB}_{\mathrm{s}} \mathrm{Ag}$ was consistently negative, while paired cytomegalovirus (CMV) titres, the Paul-Bunnell heterophil antibody test, leptospirosis complement fixation tests and an auto-antibody screen were negative. Serial blood cultures were sterile and urinalysis revealed only bilirubin. On the basis of the history, physical examination and above investigations a provisional diagnosis of posthepatitic cholestasis was made.

Because of the persistence of his pruritus and the level of hyperbilirubinaemia oral prednisolone was commenced after 10 days of conservative therapy with chlorpheniramine maleate. There was, however, no ensuing decrease in his pruritus or hyperbilirubinaemia even after intramuscular methyl prednisolone therapy had been instituted. His serum alkaline phosphatase continued to increase and a Menghini liver biopsy was accordingly performed. The appearances were those of cholestatic hepatitis and were consistent with an infective origin. Because of the firm diagnosis of infective hepatitis in his brother, therapy was continued with prednisolone for 2 weeks but, because of the unaltered hyperbilirubinaemia and his severe generalized pruritus, it was then replaced by cholestyramine. On cessation of corticosteroid therapy a pyrexia appeared, and persisted in spite of 5 days of bolus intravenous cephaloridine therapy $2 \mathrm{~g}$ daily.

The jaundice and pruritus continued in spite of cholestyramine therapy. The liver became progressively enlarged and irregular, and at this stage the gall bladder became palpable. The blood urea, which had previously remained below $8 \mathrm{mmol} / \mathrm{l}$, began to rise progressively and was followed by the 
development of hepatic failure with ascites. Abdominal paracentesis revealed serous fluid containing many adenocarcinoma cells. In view of the patient's progressive deterioration and the recent infective aetiology diagnostic laparotomy was not performed. Hepatic and renal failure progressed inexorably in spite of therapy.

At post-mortem there was considerable distension of the gall bladder and common bile duct related to an extensive carcinoma of the head of the pancreas, which on histology proved to be a primary ductal adenocarcinoma. Metastases were present in the liver, mesentery, peritoneum and omentum, and two litres of ascitic fluid were present within the abdominal cavity. The spleen, which was free from metastatic tumour, was markedly enlarged. Histological examination of the liver showed, in addition to numerous deposits of metastatic adenocarcinoma, incorporation of hepatocytes into portal tract fibrous tissue to a greater degree than would be expected from extrahepatic obstruction alone.

\section{Discussion}

Cholestasis can result from interference with bile flow at any site between the hepatic microsomes and the duodenum. Intrahepatic cholestasis with marked hyperbilirubinaemia often follows acute hepatocellular injury, as in drug-induced or viral hepatitis. If chronic cholestasis supervenes, differentiation from post-hepatic obstruction can be difficult unless percutaneous intrahepatic cholangiography or operative cholangiography is performed (Sherlock, 1966).

In the present patient the initial diagnosis of virus A hepatitis proceeding to chronic cholestasis was made on clinical and histological grounds and was corroborated by the development of a similar illness with identical histopathological findings in his brother. In the absence of a readily available reliable marker for short incubation period hepatitis no other diagnostic criteria at present exist.
Prednisolone therapy may be diagnostically helpful in patients with chronic cholestasis (Wruble et al., 1966) and may clear the jaundice where there is no extrahepatic obstruction (Sherlock, 1975). The possibility of coincidental extrahepatic obstruction was entertained when the jaundice and pruritus failed to clear with prednisolone. Parenteral corticosteroid therapy was then tried, the possibility being that the oral prednisolone was being poorly absorbed, a situation which may theoretically arise following acute viral hepatitis (Gudmand-Hözer and Söeberg, 1973), but was subsequently replaced by cholestyramine, a drug known to be effective in alleviating the pruritus of chronic cholestasis (Datta and Sherlock, 1966). The failure of this therapy, the presence of increasing hepatomegaly and then the development of a palpable gall bladder and malignant ascites made the diagnosis of extrahepatic obstruction by carcinoma certain at a time when the position was already irremediable. The dangers inherent in the use of corticosteroids in jaundiced patients and in the interpretation of a response or non-response to such therapy in patients with severe cholestatic jaundice are clear.

\section{References}

DatTA, D.V. \& Sherlock, S. (1966) Cholestyramine fof long-term relief of the pruritus complicating intrahepate cholestasis. Gastroenterology, 50, 323.

GudMAND-HözER, E. \& SöEBERG, B. (1973) Jejunal brush border disaccharidase and alkaline phosphatase activity in acute viral hepatitis. Scandinavian Journal of Gastroenterology, 8, 377.

SHERLOCK, S. (1966) Biliary secretory failure in man. The problem of cholestasis. Annals of Internal Medicine, 65, 397,

SHERLOCK, S. (1975) Diseases of the Liver and Biliary system. Fifth Edn, Blackwell Scientific Publications, Oxford London, Edinburgh and Melbourne.

Wruble, L.D., Kalser, M.H., Jones, R.H., Vloedman, D. \& BACHORIK, P. (1966) Jaundice: value of 5-day steroid test in the differential diagnosis of jaundice. Journal of the American Medical Association, 195, 184. 